\title{
Cell cycle regulation of hematopoietic stem or progenitor cells
}

\author{
Sha Hao ${ }^{1} \cdot{\text { Chen } \text { Chen }^{1} \cdot \text { Tao Cheng }}^{1,2,3}$
}

Received: 16 December 2015 / Revised: 7 March 2016 / Accepted: 7 March 2016 / Published online: 23 March 2016

(C) The Japanese Society of Hematology 2016

\begin{abstract}
The highly regulated process of blood production is achieved through the hierarchical organization of hematopoietic stem cell (HSC) subsets and their progenies, which differ in self-renewal and differentiation potential. Genetic studies in mice have demonstrated that cell cycle is tightly controlled by the complex interplay between extrinsic cues and intrinsic regulatory pathways involved in HSC self-renewal and differentiation. Deregulation of these cellular programs may transform HSCs or hematopoietic progenitor cells (HPCs) into disease-initiating stem cells, and can result in hematopoietic malignancies such as leukemia. While previous studies have shown roles for some cell cycle regulators and related signaling pathways in HSCs and HPCs, a more complete picture regarding the molecular mechanisms underlying cell cycle regulation in HSCs or HPCs is lacking. Based on accumulated studies in this field, the present review introduces the basic components of the cell cycle machinery and discusses their major cellular networks that regulate the dormancy and cell cycle progression of HSCs. Knowledge on this topic would help
\end{abstract}

Tao Cheng

chengtao@ihcams.ac.cn

Sha Hao

haosha1012@163.com

1 State Key Laboratory of Experimental Hematology, Institute of Hematology and Blood Diseases Hospital, Chinese Academy of Medical Sciences and Peking Union Medical College, Tianjin 300020, China

2 Department of Stem Cell and Regenerative Medicine, Center for Stem Cell Medicine, Chinese Academy of Medical Sciences and Peking Union Medical College, Tianjin 300020, China

3 Collaborative Innovation Center for Cancer Medicine, Tianjin 300020, China researchers and clinicians to better understand the pathogenesis of relevant blood disorders and to develop new strategies for therapeutic manipulation of HSCs.

Keywords Hematopoiesis $\cdot$ Hematopoietic stem cells . Cell cycle $\cdot$ Self-renewal $\cdot$ Quiescence

\section{Introduction}

Normal hematopoiesis is a strictly regulated process that is achieved through self-renewal of hematopoietic stem cells (HSCs), proliferation of lineage-committed hematopoietic progenitor cells (HPCs), and maturation of differentiated cells [1]. HSCs must maintain themselves at a relatively constant number throughout life by precisely balancing self-renewal and differentiation [2-4]. Most HSCs cycle infrequently and primarily reside in the G0 phase in the hypoxic bone marrow (BM) niche under homeostatic conditions [2]. However, upon cell cycle entry, HSCs give rise to progenitor populations undergoing massive proliferative expansion to meet the needs of blood cell production on a daily basis or in response to stress [5].

Cell cycle regulation of HSCs is vitally important during the progression of hematopoiesis. Hematopoiesis evolves from different anatomical sites during embryogenesis and operates through adulthood. Notably, cell cycle generally becomes more frequent as HSCs are gradually committed to lineage-restricted progenitor in adults [6, 7]. Moreover, cell cycle regulators have been shown to be important determinants of stem cell self-renewal [8, 9]. The core molecular machinery governing the mammalian cell cycle consists of a family of serine/threonine protein kinases called cyclin-dependent kinases (CDKs) [10]. Activation of $\mathrm{CDKs}$ requires binding with cyclin regulatory 
Fig. 1 The core molecular machinery on cell cycle regulation. The cell cycle processes are achieved mainly through the regulation of cyclins and cyclindependent kinases (CDKs). CDKs activation requires binding with the cyclin regulatory subunits, which are expressed in different combinations during specific phases of the cell cycle. CDK-cyclin complexes is further regulated by CDK inhibitors (CKIs), which belong to either the INK4 or CIP/KIP families, play an important role in arresting the cell cycle in G1 phase upon a variety of stimuli. Cyclin-D-CDK4/6 and cyclin E-CDK2 cooperatively phosphorylate RB family proteins, derepressing E2F to allow transcription of E2F-target genes, thereby promoting the G1/S transition

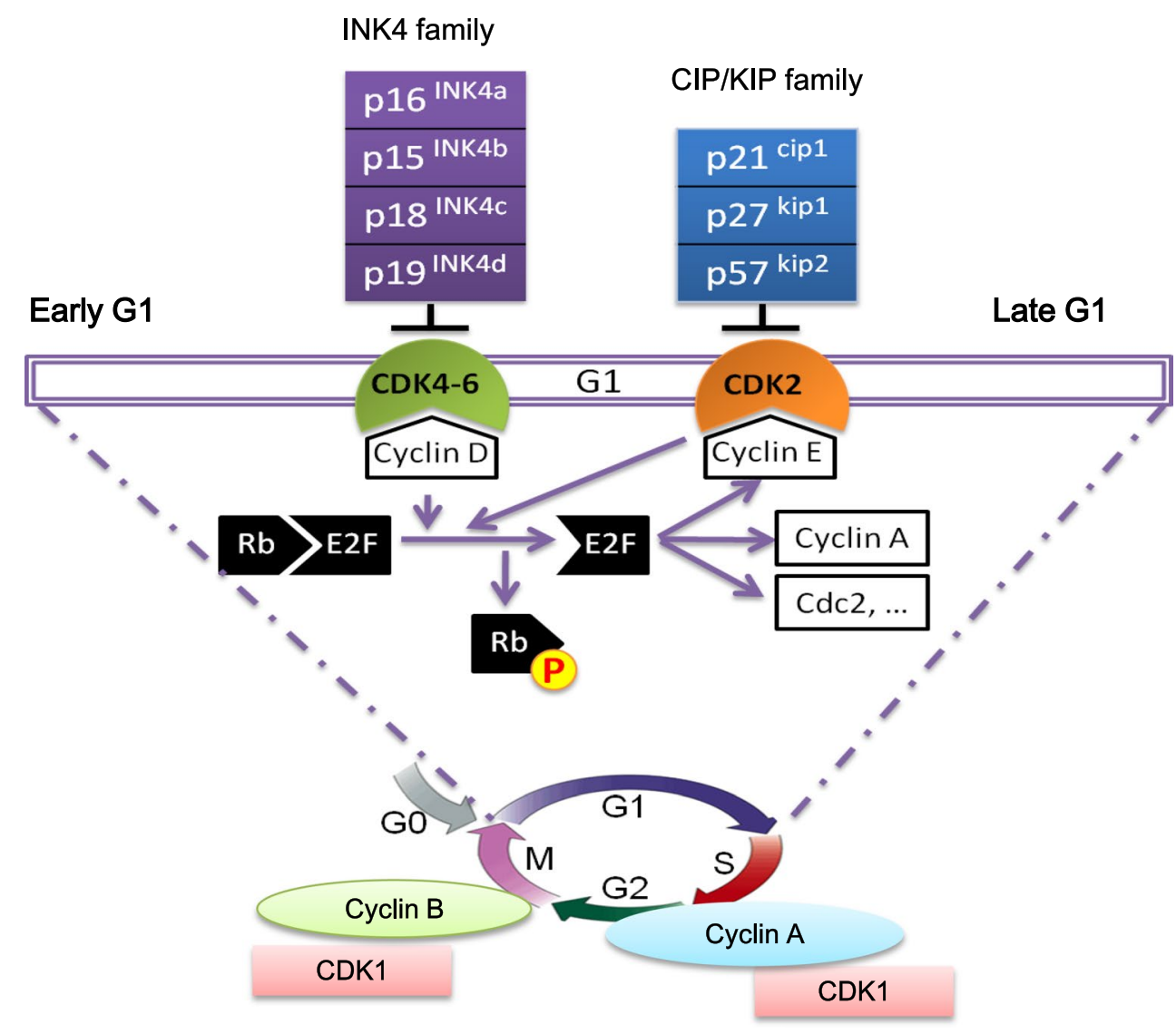

subunits. The activity of CDK/cyclin complexes is further regulated by $\mathrm{CDK}$ inhibitors (CKIs), which belong to either the INK4 or CIP/KIP families [11] (Fig. 1). Gene knockout mouse models have significantly contributed to our understanding of the physiological functions of CDKs, cyclins and CKIs in hematopoiesis. This process is also regulated by signals from the surrounding microenvironment of different organs in which hematopoiesis occurs [12]. Thus, characterization of how the microenvironmental elements, such as cytokines, and extracellular matrix (ECM), regulate HSC activity is also essential for developing new strategies for therapeutic manipulations of the stem cells.

Although the cell cycle regulation of HSCs have been reviewed previously [13-17], knowledge of the molecular mechanisms that underlies the cycling or quiescent properties of HSCs remains incomplete. The present review summarizes recent findings on this topic and focuses particularly on the impact of classical cell cycle regulators, as well as a few non-classical cell cycle regulators on hematopoietic stem and progenitor cells (HSPCs). Given the importance of proper growth control of HSCs under physiological conditions, this review may also help researchers and clinicians to better understand the pathophysiology of relevant blood disorders.

\section{An overview of hematopoiesis and cell cycle characteristics of HSCs}

The earliest hematopoiesis, referred to as "primitive" hematopoiesis, originates in the yolk sac at around embryonic day E7.5 in mice [18, 19]. The primitive wave is transitory, and at this stage erythroid progenitors are not pluripotent and do not self-renew. The subsequent wave, "definitive" hematopoiesis, occurs later in development, at different time points at different sites [1]. Definitive HSCs are found in the aorta-gonad-mesonephros (AGM) region and the placenta by E8.5 and E10, respectively, then shift to the fetal liver (FL) at E11.5, and finally the bone marrow (BM) at E16.5. The BM remains the major hematopoietic organ after birth and in adult mice [19]. During fetal life, between 95 and $100 \%$ of HSCs are actively cycling in the mouse FL, with a cell cycle transit time between 10 and $14 \mathrm{~h} \mathrm{[20].} \mathrm{Thus,} \mathrm{the} \mathrm{cell} \mathrm{cycle} \mathrm{of} \mathrm{HSCs} \mathrm{is} \mathrm{active} \mathrm{during}$ embryogenesis to ensure expansion of the stem cell pool.

In contrast, the ability of adult HSCs to reside in the quiescent state has been thought to be pivotal for maintaining the "stemness" of HSC. Irving L. Weissman's group first analyzed the proliferation and cell cycle kinetics of longterm self-renewing HSC (LT-HSC) in normal adult mice. 
They reported that approximately $5 \%$ of LT-HSCs were in S/G2/M phases of the cell cycle at any one time point and $99 \%$ of LT-HSCs divide on average every 57 days [21]. This conclusion was further supported from Trumpp group's work $[8,22]$. Computational modeling combined with functional assays suggests that dormant HSCs divide about five times over the course of a lifetime. Extrapolation of the cycling kinetics of dormant mouse HSCs to humans indicates that some human HSCs only divide about once every 18 years. Thus, quiescence or a low proliferation rate is necessary for the maintenance and self-renewal of primitive stem cells, while a high cycling rate is required for efficient generation of progenitors, and withdrawal from the cell cycle is associated with terminally differentiated cells $[6,7]$.

\section{Classical cell cycle regulators for HSC}

\section{CDKs}

Cyclin-dependent kinases, which are the master regulators of cell cycle, are activated by binding to cyclin regulatory subunits, which are expressed in different combinations during specific phases of the cell cycle.

\section{CDK4 and CDK6}

Binding to cyclin D and function in early to middle G1 phase, and this process is followed by the activation of CDK2 in complex with cyclin E during late G1. Mice deficient in CDK6 are viable and display mild defects in defined hematopoietic cell populations. In the FL of mice deficient in CDK4 or CDK6, numbers of HSCs, common lymphoid progenitors (CLPs), common myeloid progenitors (CMPs) and granulocyte-macrophage progenitors (GMPs) are all reduced, whereas double-mutant embryos undergo normal organogenesis [23]. CDK4/6 $6^{-1-}$ double knockout (DKO) mice display similar phenotypes to cyclinD $1 / 2 / 3^{-1-I-}$ triple knockout (TKO) mice, showing late embryonic lethality caused by a defect in fetal hematopoiesis, and severe dysfunction of erythropoiesis [23]. These findings indicate important roles for the cyclin DCDK4/6 complex in the cell cycle progression of fetal HSCs. Whether the cyclin D-CDK4/6 complex is involved in proliferation and maintenance of adult HSCs remains incompletely defined. John Dick's group recently reported that differential expression of CDK6 may underlie the heterogeneity of stem cell quiescence (G0) [6].

\section{$C D K 2$}

CDK2 together with cyclin E can drive cells through the G1-S transition, and with cyclin A through $\mathrm{S}$ phase.
CDK2-deficient mice are viable and have a life span of more than 2 years, suggesting that CDK2 is dispensable for the proliferation and survival of most somatic cell types. However, while CDK2 mutant mice are overtly normal, they are sterile, as CDK2 has been shown to be essential for the completion of prophase I during meiotic cell division in male germ cells [24]. Notably, no evident abnormalities were observed in HSCs from CDK2-deficient mice, indicating that other molecules (such as the CDK1-cyclin E complex as reasoned in the following section) may compensate for the loss of CDK2 [25]. Although mice lacking either CDK2 or CDK4 are viable, double knockout mutants for these two molecules are embryonically lethal at around E15 due to heart defects, indicating that CDK2 and CDK4 play synergistic roles during development $[24,26]$.

\section{CDK1}

CDK1 drives the cell cycle through $\mathrm{G} 2$ and $\mathrm{M}$ phases in association with cyclin A and cyclin B, respectively. CDK1 is the founding member of the CDK family and the only essential cell cycle CDK subfamily that drives cell mitosis [27]. In embryos lacking all interphase CDKs (CDK2, CDK3, CDK4, and CDK6), CDK1 was found to be able to bind to all cyclins (cyclin A, B, D and E), resulting in phosphorylation of retinoblastoma $(\mathrm{Rb})$ protein and the expression of genes that are regulated by the E2F transcription factors [28]. Conditional CDK1 knockout mice are not viable, and the resulting embryonic fibroblasts arrest in G2 subsequent to the induction of CDK1 loss, frequently having undergone DNA re-replication as a result of elevated CDK2-cyclin A activity. In contrast, embryos lacking CDK1 fail to develop to the morula and blastocyst stages [28].

\section{Cyclins}

\section{Cyclin D}

The D-cyclin family includes cyclin D1 (CCND1), cyclin D2 (CCND2) and cyclin D3 (CCND3), all of which expressed in HSCs [9]. D-type cyclins are thought to be the sensors of extracellular environment that link mitogenic signaling pathways to the core machinery of the cell cycle. It has been reported that mice lacking all three D-type cyclins manifest a significant reduction of HSPC number accompanied by a loss of reconstitution capacity, and die in late embryogenesis with severe anemia [29]. In contrast, mice deficient in a single cyclin D show few hematopoietic defects, likely due to functional redundancy of the cyclin D family in hematopoiesis [30]. 


\section{Cyclin A}

Two A-type cyclins, A1 and A2, are expressed in mammals [31]. Using conditional knockout mice, Kalaszczynska et al. found that cyclin A function was essential for cell cycle progression of HSCs and embryonic stem cells [32]. Conditional inactivation of the cyclin $\mathrm{A} 2$ gene in mice reduced numbers of HSCs and CMPs, and significantly impaired the reconstitution ability of cyclin A2-deficient BM cells [32]. Cyclin A was shown to be expressed at high levels whereas cyclin $\mathrm{E}$ was nearly absent in the hematopoietic lineage, thereby suggesting that cyclin A-CDK complex may play a more crucial role than cyclin E-CDK complex in HSCs.

\section{Cyclin E}

Proliferating cells express two types of cyclin E, cyclin E1 (CCNE1) and cyclinE2 (CCNE2). Mice lacking cyclin E1 or cyclin E2 have a normal life span without overt abnormalities. Although cyclin E2-deficient mice were viable, the mutant male's fertility was reduced. Double mutants of cyclins E1 and E2 showed severely impaired endoreplication of megakaryocytes and trophoblast giant cells, and died around midgestation during embryogenesis [33, 34]. As discussed above, hematopoiesis in cyclin E-deficient mice appears relatively normal, which may be due to the complementary effect of other CDKs [35].

\section{Cyclin B}

There are three B-type cyclins, cyclins B1, B2 and B3 in mammalian cells. HSCs of mice lacking cyclin B1 remain largely uncharacterized, but these animals die in utero at an early stage. In contrast, cyclin B2-deficient mice develop normally and do not manifest notable abnormalities [36]. Since cyclin B3-null mice have not been generated, the functions of this cyclin in hematopoiesis remain to be defined.

\section{CKIs}

CKIs are proteins that interact with cyclin-CDK complex to block kinase activity, play an important role in arresting the cell cycle in G1 phase upon a variety of stimuli. Given their vital roles in cell cycle control of HSPCs, they are involved in homeostasis of normal hematopoiesis as well as disordered hematopoiesis $[15,16]$. There are two major CKI families: the INK4 (Inhibitor of CDK4) family and the CIP/KIP (CDK2 interacting protein/kinase inhibition protein) family. The INK4 family consisted of CKIs $\mathrm{p} 16^{\mathrm{INK} 4 \mathrm{a}}, \mathrm{p} 15^{\mathrm{INK} 4 \mathrm{~b}}, \mathrm{p} 18^{\mathrm{INK} 4 \mathrm{c}}$, and p19 $9^{\mathrm{INK} 4 \mathrm{~d}}$. Biochemically, they all play roles as antagonists of the cyclin D-CDK4/6 complex, thereby blocking phosphorylation of $\mathrm{Rb}$ family members and subsequent entry into $\mathrm{S}$ phase. The CIP/KIP family includes $\mathrm{p} 21^{\mathrm{CIP}}, \mathrm{p} 27^{\mathrm{KIP} 1}$ and $\mathrm{p} 57^{\mathrm{KIP} 2}$, which controls entry into $S$ phase by inhibiting the activity of the cyclin E-CDK2 complex. Interestingly, p21 and p27 can also facilitate the activation of cyclin D-CDKs, and are shown to control the pool size of HSPCs by maintaining the quiescence $[37,38]$.

p16 ${ }^{I N K 4 a}$

p16 is expressed at a higher level in $\mathrm{CD} 34^{+}$cells than $\mathrm{CD} 34^{-}$cells, and its expression is progressively decreased during the commitment of HSC into progenitors [39], thus it may play a role in mitotic quiescence (or dormancy) of HSCs. However, since $\mathrm{p} 16^{-/-}$mice do not exhibit any apparent abnormality in hematopoiesis, it is believed that p16 is dispensable for the maintenance of HSC quiescence, at least in young mice [40]. Interestingly, the expression of p16 increases with age or in response to stresses, such as reactive oxygen production. Absence of p16 can ameliorate the defects in repopulating activity and apoptosis in HSCs that are associated with aging [41]. Furthermore, activation of the p16-CDK4/CDK6-Rb signaling pathway has been shown to contribute to defective maintenance of stem cells in response to elevated intracellular reactive oxygen species (ROS), a situation associated with aging [42]. In summary, the physiological impact of aging on stem cells can be alleviated by inhibition of p16 in aged cells.

$p 15^{I N K 4 b}$

Unlike the expression of p16, p15 is undetected in CD34 cells and its expression does not increase during myeloid differentiation [43]. $\mathrm{p} 15^{-1-}$ mice are viable and exhibit no apparent defects in HSCs function, except for an enhancement of the proliferation of CMPs [44]. However, it has been reported that 15 is induced by TGF- $\beta 1$ [45]; thus, the role of p15 in HSCs remains to be further defined.

$p 18^{I N K 4 c}$

p18 is expressed in hematopoietic cells including quiescent HSCs [9]. p18 null mice develop organomegaly owing to increased cellularity, with an increased incidence of tumorigenesis, especially after exposure to carcinogens [46]. Deficiency of p18 results in an expansion of stem and progenitor cells, which show a competitive advantage in serial bone marrow transplantation assays compared with that of normal controls [47, 48]. Among all the CKIs, p18 appears to have the strongest impact on HSC self-renewal in mice [49]. Regarding its mechanism, it has been proposed that HSCs have a shorter G1 phase due to a lower cyclin D/ 
CDK4 threshold activity. According to this model, mutant cells transit more rapidly through late $\mathrm{G} 1$, and thus would be less exposed to differentiation-inducing cytokine stimuli, resulting in an increased capacity for self-renewal [50]. However, the significant effect of p18 absence on HSC cannot be solely explained by its classical role in cell cycle regulation, as deletion of p18 did not significantly affect the cell cycle profile of HSCs despite its profound effect on HSC engraftment and reconstitution in recipient mice [49]. Interestingly, our recent work demonstrated a specific role of small molecule inhibitors for p18 protein on HSC expansion, indicating the potential value of targeting p18 for therapeutic expansion of HSC $[49,51]$.

$p 19^{I N K 4 d}$

p19-deficient mice survive with a normal life span and do not show increased susceptibility to the spontaneous tumors. It has been reported that p19 regulates the maturation of megakaryocytes in humans and mice [52]. However, the potential involvement of p19 in HSC regulation still needs to be studied [53].

$p 21^{C I P}$

p21 was the first CKI whose deletion was reported to result in the exhaustion of HSCs. It was initially indicated to be a key molecule that restricts the entry of HSCs into the cell cycle in mice with the 129/sv background, imposing limits on their pool size and preventing their exhaustion under prolonged stress [37]. However, other studies [54] have suggested that the expression level of p21 was not very high in $\mathrm{CD} 34^{+}$cells, and p21-null mice do not seem to exhibit an altered hematologic profile under homeostatic conditions in the mice with the C57/B6 background. However, when BM cells were transplanted after 2 Gy of irradiation, p21 deficiency resulted in a more than fourfold reduction in competitive repopulation index. More recent data suggest that the role of p21 is mainly restricted to genotoxically stressed HSCs. Unlike other members of the CKI families, p21 is a p53 target gene and is a key mediator of p53-mediated G1 arrest [55]. In response to DNA damage provoked by telomerase dysfunction, CD34 ${ }^{-}$KLSHSCs are shown to up-regulate $\mathrm{p} 21$, detrimentally affecting their function. In this setting, HSC self-renewal is rescued by p21 deletion [56]. The significance of $\mathrm{p} 21$ in regulating HSC cell cycle activity may thus be more evident under stress.

$p 27^{K I P I}$

p27 interacts with similar CDKs as p21. Unlike p21, the expression of $\mathrm{p} 27$ is independent of $\mathrm{p} 53$. Mice lacking $\mathrm{p} 27$ have different phenotypes compared with p21-deficient mice, such as increased body sizes, and hyperplasia of most organs including hematopoietic organs. In striking contrast to $\mathrm{p} 21^{-I-}$ mice, $\mathrm{p} 27^{-1-}$ mice do not have altered number, cycling or self-renewal of HSCs, but show a significant increase in the proliferation rate and pool size of the progenitor compartments [57]. These progenitor cells in p $27^{-1-}$ mice are more proliferative than $\mathrm{p} 27^{+/+}$progenitor cells. As a result, progenitor cells from $\mathrm{p} 27^{-/-}$mice have the ability to expand and regenerate after serial transplantation, thereby establishing an intriguing paradigm in which stem cell repopulation efficiency can be increased without increased stem cell number [38]. Thus, p27 negatively regulates the hematopoietic progenitor pool without a significant impact on HSC abundance. However, given the presence of p27 in both HSC and HPC compartments, a role for this factor in HSCs cannot be ruled out. It has been reported that p27 was able to interact not only with p57, but also with the Myc-Max-Mad transcription factors, and p27/Mad1 double mutants display increased HSC number $[8,58,59]$. More details regarding the role of $\mathrm{p} 27$ in HSCs are discussed in the following section.

$p 57^{K I P 2}$

p57 transcripts and protein are highly expressed in the Thy $1.1^{\text {int }}$ Flk2 ${ }^{-}$LSK [9] and $\mathrm{CD}^{-} 4^{-} \mathrm{MPL}^{+} \mathrm{LSK}$ primitive, quiescent HSCs [60]. This expression progressively declines during differentiation toward less self-renewing multipotent progenitors (MMPs) [9, 61, 62]. Matsumoto and colleagues reported that p57-deficient mice die immediately after birth due to severe developmental defects, with a significant reduction in numbers of hematopoietic progenitors $\left(\mathrm{CD} 34^{+} \mathrm{LSK}, \mathrm{CD} 150^{+} \mathrm{CD} 48^{-} \mathrm{LSK}\right)$ and primitive $\mathrm{CD}^{-} 4^{-} \mathrm{LSK}$ HSCs, size of the quiescent HSC pool and reconstitution ability of HSCs after transplantation [63]. The dependency of HSCs on p57 was specific to this CKI because mutants of the other two CIP/KIP members, p21 and p27, did not show defective repopulating potential, which has been confirmed in earlier reports $[38,64]$. Additional ablation of p27 in p57-deficient HSCs results in pronounced defects in maintenance of HSC quiescence. Interestingly, knock-in of p27 at p57 locus corrects the abnormalities of HSCs in p57-deficient mice [63]. Moreover, Zou et al. reported that p57 contributes to the maintenance of HSC quiescence, and p27 compensates for p57 function in HSCs with a p57 deletion. An association between p57, p27, and Hsc70 was found to maintain the cytoplasmic localization of the Hsc70/cyclin D1 complex and regulate the cell cycle entry of HSCs. Their findings provide new insights into the physiological function of p57 and p27 in HSCs and have implications for the roles of CDK inhibitors in the maintenance of tissue homeostasis [59]. 
Fig. 2 Hematopoietic stem cells maintained by the CDK inhibitors. Hematopoietic stem cells (HSCs) maintain themselves with a relatively constant number by precisely balancing self-renewal and differentiation. CDK inhibitors (CKIs) play distinct roles in HSPCs. Of all the CKIs, p18 appears to have the strongest inhibitory effect on HSC self-renewal. Whereas, p21 could prevent exhaustion of HSCs, p57 contributes to the maintenance of HSC quiescence. Unlike p21, p27 alone does not overly affect self-renewal of HSCs, but its absence results in a significant increase in the pool size of the progenitor compartments. p16 appears to be more critical for the maintenance of HSC function during aging

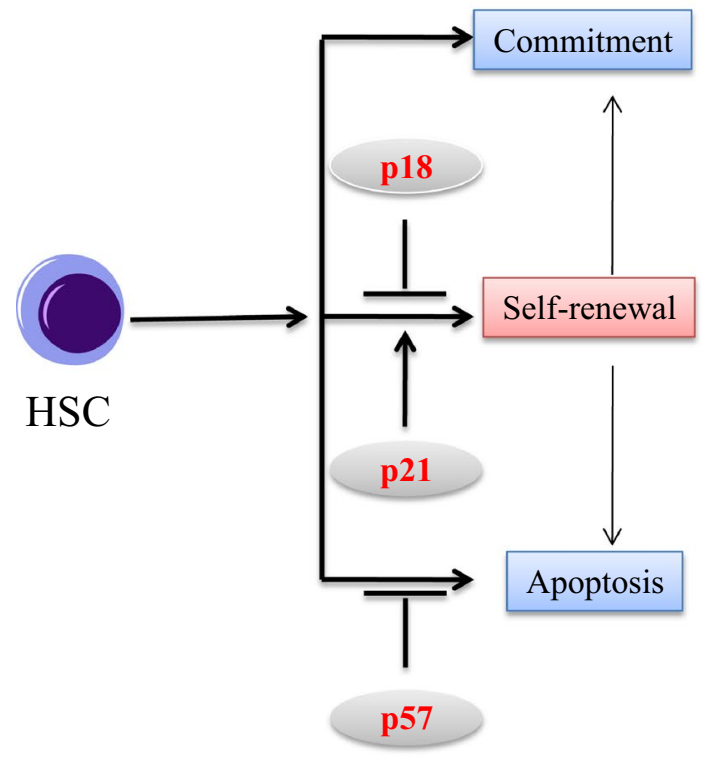

CKIs thus play distinct roles in HSPCs. Of all the CKIs, p18 appears to have the strongest inhibitory effect on HSC self-renewal whereas p21 could prevent exhaustion of HSCs. p57 contributes to the maintenance of HSC quiescence. Unlike p21, p27 alone does not overtly affect selfrenewal of HSCs, but its absence results in a significant increase in the pool size of the progenitor compartments. p16 appears to be more critical for the maintenance of HSC function during aging (Fig. 2).

\section{The transcription factors affecting HSC cell cycle}

The HSC cell cycle is also regulated by many transcription factors, such as c-Myb, GATA-2, Gli-1 and the Hox family. We provide few examples here.

\section{c-Myb}

c-Myb is a DNA binding transcription factor that controls differentiation and proliferation in hematopoietic and other cell types, and is latent in regulation during the cell cycle. c-Myb interacts with cyclin D1 and CDK4/CDK6 [65, 66]. In addition, c-Myb has been shown to regulate the CCNB1 and CCNE1 genes, which encode Cyclin B1 and Cyclin E1, respectively [67, 68]. c-Myb is highly expressed in immature and proliferative cellular stages, and its expression is switched off during maturation of the hematopoietic lineage. It is thus considered a critical regulator of proliferation and differentiation of HSCs. c-Myb-deficient mice are embryonic lethal by around E15.5 due to an abnormal definitive hematopoiesis. Conditional disruption of $\mathrm{c}-\mathrm{Myb}$ resulted in dramatic reductions in CMP, GMP and MEP, leading to a reduction of neutrophils, basophils, monocytes and platelets in peripheral blood [69]. c-Myb promotes the growth of HSCs, probably through the induction of c-Myc and up-regulating expression of c-kit and Flt3. Scott A. Ness reported that c-Myb could actively reposition, binding to different gene promoters during the specific cell cycle phases. Liu D et al. confirmed the critical role of c-Myb in promoting Erbin transcription in G2/M phase and also predict the function of Erbin in cell cycle progression [70].

\section{GATA-2}

Transcriptional profiling has shown high expression of GATA-2 in quiescent hematopoietic cells, but no causal relationship between GATA-2 level and quiescence has been clearly defined. GATA-2 ${ }^{-1-}$ mice die at E11.5 with defects in the development and/or maintenance of HSCs [71]. However, the specific functional roles of GATA-2 in HSC growth are still controversial. It is reported that GATA-2 suppresses the growth of HSCs using a ligandinducible chimeric form of GATA-2(GATA-2/ER) [72]. In contrast, another group shows that GATA-2 promotes the growth of HSCs and the conflicting data could result from a biologically significant difference between wild-type GATA-2 and GATA-2/ER [73]. In addition, Persons et al. report that wild-type GATA-2 can inhibit the growth of 
HSCs [74]. Thus, whether GATA-2 suppresses or promotes growth of HSCs remains unclear.

\section{Gfi-1}

Growth factor independent 1 (Gfi-1) is a zinc finger transcriptional repressor, which plays a role in the regulation of development of adult HSPCs. Orkin's group showed that although Gfi-1 and Bmi-1 had similar functions in lymphomagenesis, which promotes the proliferation of lymphoid cells, their roles in HSCs differ. Different from the impaired proliferation and maintenance of HSCs in Bmi- $1^{-1-}$ mice, the LT-HSCs in bone marrow of $\mathrm{Gfi}^{-1-}$ mice were increased. Their study also suggested that the p $21^{\mathrm{CIP} 1}$ may be one mediator of Gfi-1 in HSCs given the observation that $\mathrm{p} 21^{\mathrm{CIP} 1}$ was markedly down-regulated in Gfi- $1^{-/}$HSCs [75]. Both Orkin's and Zeng's groups found significant decreases of the short-term HSCs (ST-HSCs) and MPPs in Gfi-1 $1^{-1-}$ bone marrow, while they showed differences regarding the total HSCs and LT-HSCs. Specifically, Zeng et al. [76] noted a decrease of LSK and LTHSCs in Gfi-1-null mice, which was opposite to the result from Orkin's group [75]. These differences may be caused by different genetic backgrounds of the mice they used or by biases in fluorescence-activated cell sorting (FACS) and cell counts in defining these cell populations. Nevertheless, both groups observed the same functional consequences such as compromised short-term radioprotection capacity, indicating defects in the ST-HSC, MPP and CMP populations, and decreased long-term and competitive reconstitution capacity of HSCs in the absence of Gfi-1.

\section{HOX family}

Several homeobox transcription factors, such as HOXB3 and HOXB4, have been reported in regulation of hematopoiesis. In support of this, studies have showed that overexpression of HOXB4 strongly enhances HSCs expansion [77], and both HOXB4 and HOXB7 were reported to induce expansion of HSCs in vitro [78, 79]. Double HOXB3 and HOXB4 mutant mice have defects in endogenous hematopoiesis with reduced cellularity in hematopoietic organs and a diminished number of hematopoietic progenitors without perturbing their lineage commitment. This study demonstrated that both HOXB4 and HOXB3 are required for the maximal proliferative response of HSCs [80]. Weissman's group recently identified a single gene, HOXB5, the expression of which is limited to longterm HSC(LT-HSC), and showed the long-term reconstitution capacity after primary and secondary transplantation. Notably, only $7-35 \%$ of various previously defined immunophenotypic HSCs are LT-HSCs [81].

\section{The cytokines regulating HSC cell cycle}

\section{TPO}

Signaling by TPO and its receptor Mpl has been shown to be crucial for maintaining quiescence and self-renewal capacity of HSCs since the loss of TPO signaling was associated with bone marrow failure and thrombocytopenia [82]. It was thought that TPO's role in the regulation of HSCs only begin postnatally, as $\mathrm{Mpl}^{-1-}$ and $\mathrm{TPO}^{-1-}$ fetal livers are found to have the same numbers of E12.5 progenitor cells as found in wild-type mice, and an equivalent ability to repopulate the bone marrow with E14.5 fetal liver cells after transplant [83, 84]. Quiescent HSCs express Mpl and reside in the niche that is close to TPO-producing osteoblastic cells. It was reported that Mpl-deficient mice manifest reduced number of HSCs with severely impaired activity in competitive reconstitution assays [85]. Activation of TPO-Mpl signaling results in up-regulation of p57 expression in HSCs, whereas elimination of TPO or Mpl activation results in the opposite effects $[60,84]$. The TPO-Mpl pathway works in adult HSCs but not in embryonic HSCs, and the TPO-Mpl-p57 axis, therefore, may be a crucial signaling pathway for regulation of stemness and quiescence of adult HSCs. The link between this pathway and the cell cycle machinery of HSCs appears to be mediated, at least in part, by the regulation of cell cycle inhibitors p57 in HSCs $[60,84]$.

\section{TGF- $\beta$}

Transforming growth factor $\beta$ (TGF- $\beta$ ) is one of the best known negative regulators of hematopoiesis [86, 87]. Targeting TGF- $\beta$ signaling resulted in embryonic lethality in mice [88]. Using the conditional knockout strategy, deficiency in TGF- $\beta$ receptor I caused no defect in HSC quiescence or in maintenance of the HSC pool [89]. However, mice deficient in TGF- $\beta$ type II receptor showed reduced repopulating activity. Interestingly, TGF- $\beta 1$ is able to stimulate myeloid-biased HSCs (My-HSCs) to proliferate whereas exerts its inhibitory role on lymphoid-biased HSCs (Ly-HSCs) [90]. One of the molecular mechanisms governing the proliferative response of My-HSCs to TGF- $\beta 1$ appears to be down-regulation of p18 and p19 [90]. Recently, Li L group reported that ablation of megakaryocytes (MK) led to activation of quiescent HSCs and increased HSC proliferation, due to reduced levels of biologically active TGF- $\beta 1$ protein. TGF- $\beta 1$ injection restored HSC quiescence, and TGF- $\beta 1$ knockout in MK also resulted in a decrease in quiescent HSCs [91]. These findings indicate that the TGF- $\beta$ signaling pathway plays an important role in the maintenance of quiescent HSCs. 


\section{Other non-classical regulators for HSC cell cycle}

\section{MicroRNAs}

MicroRNAs (miRNAs) are a class of noncoding RNAs interfering with gene regulation, which can regulate hematopoiesis by interactions with multiple target genes. MicroRNAs may in fact be powerful HSC regulators, but their functions are poorly understood. It has been reported that miR-29a is highly expressed in HSCs and down-regulated in hematopoietic progenitors, which can promote progenitor proliferation by expediting $\mathrm{G} 1$ to $\mathrm{S} / \mathrm{G} 2$ cell cycle transitions. As a result, ectopic expression of miR-29a can induce aberrant self-renewal capacity in hematopoietic progenitors, biased myeloid development, and acute myeloid leukemia (AML) [92]. Hu and colleagues observed decreased

Table 1 Cell cycle regulators on HSPCs

\begin{tabular}{|c|c|c|c|}
\hline & Genes & Phenotypes & References \\
\hline \multicolumn{4}{|c|}{ The classical cell cycle regulators } \\
\hline \multirow[t]{5}{*}{ CDKs } & $\mathrm{CDK}^{-1-}$ & Reduction of CLP, CMP and GMP & [23] \\
\hline & CDK4/6 DKO & Defect in fetal hematopoiesis & [23] \\
\hline & $\mathrm{CDK} 2^{-l-}$ & Viable, but sterile & {$[24,25]$} \\
\hline & CDK2/4DKO & Embryonically lethal & {$[24,26]$} \\
\hline & $\mathrm{CDK} 1^{-1-}$ & Not viable & [28] \\
\hline \multirow[t]{7}{*}{ Cyclins } & Cyclin D1/2/3 TKO & Reduction of HSPC, die at late embryogenesis with severe anemia & [29] \\
\hline & Cyclin $\mathrm{D} 1^{-l-}$ & Little hematopoietic defects & [30] \\
\hline & $\operatorname{Cyclin}^{-l-}$ & Reduction of HSCs and CMPs & [32] \\
\hline & Cyclin $E 2^{-1-}$ & Viable, but reduced fertility & [33] \\
\hline & Cyclin E1/2 DKO & Embryonically lethal & {$[33,34]$} \\
\hline & Cyclin $\mathrm{B} 1^{-/-}$ & Embryonically lethal & [36] \\
\hline & Cyclin $B 2^{-l-}$ & Normal & [36] \\
\hline \multirow{3}{*}{$\begin{array}{l}\text { CKIs } \\
\text { INK4 family }\end{array}$} & $\mathrm{p} 16^{\mathrm{INK} 4 \mathrm{a}-l-}$ & Defects in HSC with age and stress & {$[40,41]$} \\
\hline & $\mathrm{p} 15^{\mathrm{INK} 4 \mathrm{~b}-/-}$ & Enhanced proliferation of CMPs & [44] \\
\hline & $\mathrm{p} 18^{\mathrm{INK} 4 \mathrm{c}-1-}$ & Increased HSC self-renewal & {$[47,48]$} \\
\hline \multirow{3}{*}{$\begin{array}{l}\text { CKIs } \\
\text { CIP/KIP family }\end{array}$} & $\mathrm{p} 21^{\mathrm{CIP}-1-}$ & Exhaustion of HSC activity & [37] \\
\hline & $\mathrm{p} 27^{\mathrm{KIP} 1-/-}$ & Enlarged progenitor pool & [57] \\
\hline & $\mathrm{p} 57^{\mathrm{KIP} 2-1-}$ & Decreased HSC quiescence & [63] \\
\hline \multicolumn{4}{|c|}{ The non-classical cell cycle regulators } \\
\hline \multirow[t]{11}{*}{ Transcription factors } & $\mathrm{c}-\mathrm{Myb}^{-/-}$ & $\begin{array}{l}\text { Embryonically lethal } \\
\text { Dramatic reductions in CMP, GMP and MEP }\end{array}$ & [69] \\
\hline & GATA-2 ${ }^{-1-}$ & Embryonically lethal & [71] \\
\hline & & Suppressed growth of HSC & [72] \\
\hline & & Enhanced growth of HSC & [73] \\
\hline & $\mathrm{Gfi}_{-1}^{-1-}$ & Increased LT-HSC & [75] \\
\hline & & Decreased ST-HSC and MPP & {$[75,76]$} \\
\hline & & Decreased LT-HSC & [76] \\
\hline & HoxB4 & Enhanced HSC expansion & {$[77,78]$} \\
\hline & HoxB7 & Enhanced HSC expansion & [79] \\
\hline & HoxB3 & Decreased HSC proliferation & [80] \\
\hline & HoxB5 & Enhanced LT-HSC & [81] \\
\hline \multirow[t]{3}{*}{ Cytokines } & $\mathrm{Mpl}^{-1-}$ & Reduced number of HSC & [85] \\
\hline & TGF- $\beta$ & Embryonically lethal & [88] \\
\hline & TGF- $\beta \mathrm{RI}^{-1-}$ & No defects in HSC & [89] \\
\hline \multirow[t]{3}{*}{ MicroRNAs } & miR-29a & Enhanced proliferation of HSPC & {$[92,93]$} \\
\hline & $\operatorname{miR}-33$ & Enhanced HSCs self-renewal & [94] \\
\hline & miR-126 & Decreased HSC proliferation & {$[95,96]$} \\
\hline
\end{tabular}

$D K O$ double knockout, TKO triple knockout 
numbers of HSPCs, decreased HSC self-renewal, and increased HSC cell cycling and apoptosis using homozygous deletion of the miR-29a/b-1, mainly due to loss of miR-29a. Their work also suggested that Dnmt3a may be a target gene of miR-29, thus contributing to the phenotype of miR-29a/b-1-null HSPCs through negative regulation [93]. What is more, other microRNAs can work through different ways, such as miR-33 can control HSCs selfrenewal by mediating the down-regulation of p53 [94], and miR-126 can arrest cell cycle progression of HSC in vitro and in vivo by PI3 K/AKT/GSK3 pathway, increasing HSC proliferation without inducing exhaustion $[95,96]$.

\section{ECM}

HSCs receive critical signals for proliferation and differentiation from stromal cells and extracellular matrix $(\mathrm{ECM})$ in bone marrow (BM) niche. ECM is composed of a variety of molecules such as fibronectin (FN), collagens, laminin, and proteoglycans [97]. In which, FN, collagens, and laminin are ligands for integrins that not only control the anchorage and migration of HSCs, but also activate signal transduction pathways in these cells. The interaction of HSCs with FN was also reported to enhance the growth and survival of HSCs. In long-term BM cultures, HSCs preferentially reside in the adherent cell layers. Blood cell formation in these cultures is completely blocked by the inhibition or specific modulation of interactions between stromal and hematopoietic cells, suggesting the importance of these interactions. As an example of one mechanism responsible for this effect, Bernstein et al. showed that the Notch transmembrane receptors on HSCs were activated by their ligands (Jagged 1 or Jagged 2) on stromal cells, promoting self-renewal of HSCs [98]. A recent study showed that Notch receptor ligand engagement maintains hematopoietic stem cell quiescence and niche retention [99].

\section{Concluding remarks}

Precise cell cycle regulation of HSCs is an essential aspect of normal development and adult homeostasis, and dysregulation of this process is one of the hallmarks of HSC-derived diseases. Understanding how to distinguish the self-renewal property of normal HSC versus their malignant counterpart represents a key challenge in HSC biology, as well as in relevant clinical issues. While the relationship between cell cycle regulation and HSC self-renewal has been elucidated in part, as reviewed here (Figs. 1, 2; Table 1), much work remains to be done to fully understand how the regulatory networks control cell cycle activity of HSC in both normal and diseased conditions through the development to a lifespan of an organism, how multiple signaling pathways cooperate to precisely regulate the proper function of HSC, and more importantly how to manipulate these mechanisms to develop novel therapeutics of HSC for the treatment of relevant diseases.

Acknowledgments This work was supported by Grants from the National Natural Science Foundation of China (81421002, 81300374, $81430004)$, the Ministry of Science and Technology of China (2011CB964801, 2013CB966902).

Compliance with ethical standards

Conflict of interest The authors declare no conflict of interest.

\section{References}

1. Jagannathan-Bogdan M, Zon LI. Hematopoiesis. Development. 2013;140(12):2463-7.

2. Seita J, Weissman IL. Hematopoietic stem cell: self-renewal versus differentiation. Wiley Interdiscip Rev Syst Biol Med. 2010;2(6):640-53.

3. Giebel B, Bruns I. Self-renewal versus differentiation in hematopoietic stem and progenitor cells: a focus on asymmetric cell divisions. Curr Stem Cell Res Ther. 2008;3(1):9-16.

4. Weiss CN, Ito K. DNA damage: a sensible mediator of the differentiation decision in hematopoietic stem cells and in leukemia. Int J Mol Sci. 2015;16(3):6183-201.

5. Cheung TH, Rando TA. Molecular regulation of stem cell quiescence. Nat Rev Mol Cell Biol. 2013;14(6):329-40.

6. Laurenti E, et al. CDK6 levels regulate quiescence exit in human hematopoietic stem cells. Cell Stem Cell. 2015;16(3):302-13.

7. Bradford GB, et al. Quiescence, cycling, and turnover in the primitive hematopoietic stem cell compartment. Exp Hematol. 1997;25(5):445-53.

8. Tesio M, Trumpp A. Breaking the cell cycle of HSCs by p57 and friends. Cell Stem Cell. 2011;9(3):187-92.

9. Passegue E, et al. Global analysis of proliferation and cell cycle gene expression in the regulation of hematopoietic stem and progenitor cell fates. J Exp Med. 2005;202(11):1599-611.

10. Aleem E, Arceci RJ. Targeting cell cycle regulators in hematologic malignancies. Front Cell Dev Biol. 2015;3:16.

11. Aleem E, Kaldis P. Mouse models of cell cycle regulators: new paradigms. Results Probl Cell Differ. 2006;42:271-328.

12. Wilson A, Trumpp A. Bone-marrow haematopoietic-stem-cell niches. Nat Rev Immunol. 2006;6(2):93-106.

13. Pietras EM, Warr MR, Passegue E. Cell cycle regulation in hematopoietic stem cells. J Cell Biol. 2011;195(5):709-20.

14. Siu KT, Minella AC. Developing a systems-based understanding of hematopoietic stem cell cycle control. Adv Exp Med Biol. 2014;844:189-200.

15. Cheng T, Scadden DT. Cell cycle entry of hematopoietic stem and progenitor cells controlled by distinct cyclin-dependent kinase inhibitors. Int J Hematol. 2002;75(5):460-5.

16. Cheng T. Cell cycle inhibitors in normal and tumor stem cells. Oncogene. 2004;23(43):7256-66.

17. Boyer MJ, Cheng T. The CDK inhibitors: potential targets for therapeutic stem cell manipulations? Gene Ther. 2008;15(2):117-25.

18. Palis J, et al. Development of erythroid and myeloid progenitors in the yolk sac and embryo proper of the mouse. Development. 1999;126(22):5073-84. 
19. Medvinsky A, Rybtsov S, Taoudi S. Embryonic origin of the adult hematopoietic system: advances and questions. Development. 2011;138(6):1017-31.

20. Nygren JM, Bryder D, Jacobsen SE. Prolonged cell cycle transit is a defining and developmentally conserved hemopoietic stem cell property. J Immunol. 2006;177(1):201-8.

21. Cheshier SH, et al. In vivo proliferation and cell cycle kinetics of long-term self-renewing hematopoietic stem cells. Proc Natl Acad Sci USA. 1999;96(6):3120-5.

22. Wilson A, et al. Hematopoietic stem cells reversibly switch from dormancy to self-renewal during homeostasis and repair. Cell. 2008;135(6):1118-29.

23. Malumbres M, et al. Mammalian cells cycle without the D-type cyclin-dependent kinases Cdk4 and Cdk6. Cell. 2004;118(4):493-504.

24. Jayapal SR, et al. Hematopoiesis specific loss of Cdk2 and Cdk4 results in increased erythrocyte size and delayed platelet recovery following stress. Haematologica. 2015;100(4):431-8.

25. Aleem E, Kiyokawa H, Kaldis P. Cdc2-cyclin E complexes regulate the G1/S phase transition. Nat Cell Biol. 2005;7(8):831-6.

26. Berthet $\mathrm{C}$, et al. Combined loss of $\mathrm{Cdk} 2$ and $\mathrm{Cdk} 4$ results in embryonic lethality and $\mathrm{Rb}$ hypophosphorylation. Dev Cell. 2006;10(5):563-73.

27. Malumbres M, Barbacid M. Cell cycle, CDKs and cancer: a changing paradigm. Nat Rev Cancer. 2009;9(3):153-66.

28. Santamaria D, et al. Cdk1 is sufficient to drive the mammalian cell cycle. Nature. 2007;448(7155):811-5.

29. Kozar K, et al. Mouse development and cell proliferation in the absence of D-cyclins. Cell. 2004;118(4):477-91.

30. Fantl V, et al. Mice lacking cyclin D1 are small and show defects in eye and mammary gland development. Genes Dev. 1995;9(19):2364-72.

31. Nieduszynski CA, Murray J, Carrington M. Whole-genome analysis of animal A- and B-type cyclins. Genome Biol. 2002;3(12):RESEARCH0070.

32. Kalaszczynska $\mathrm{I}$, et al. Cyclin A is redundant in fibroblasts but essential in hematopoietic and embryonic stem cells. Cell. 2009;138(2):352-65.

33. Geng $\mathrm{Y}$, et al. Cyclin $\mathrm{E}$ ablation in the mouse. Cell. 2003;114(4):431-43.

34. Parisi T, et al. Cyclins E1 and E2 are required for endoreplication in placental trophoblast giant cells. EMBO J. 2003;22(18):4794-803.

35. Duronio RJ, Xiong Y. Signaling pathways that control cell proliferation. Cold Spring Harb Perspect Biol. 2013;5(3):a008904.

36. Brandeis $\mathrm{M}$, et al. Cyclin B2-null mice develop normally and are fertile whereas cyclin B1-null mice die in utero. Proc Natl Acad Sci USA. 1998;95(8):4344-9.

37. Cheng $\mathrm{T}$, et al. Hematopoietic stem cell quiescence maintained by p21cip1/waf1. Science. 2000;287(5459):1804-8.

38. Cheng T, et al. Stem cell repopulation efficiency but not pool size is governed by p27(kip1). Nat Med. 2000;6(11):1235-40.

39. Furukawa $Y$, et al. Lineage-specific regulation of cell cycle control gene expression during haematopoietic cell differentiation. Br J Haematol. 2000;110(3):663-73.

40. Serrano M, et al. Role of the INK4a locus in tumor suppression and cell mortality. Cell. 1996;85(1):27-37.

41. Janzen V, et al. Stem-cell ageing modified by the cyclin-dependent kinase inhibitor p16INK4a. Nature. 2006;443(7110):421-6.

42. Ito $\mathrm{K}$, et al. Regulation of oxidative stress by ATM is required for self-renewal of haematopoietic stem cells. Nature. 2004;431(7011):997-1002.

43. Teofili L, et al. Expression of p15INK4B in normal hematopoiesis. Exp Hematol. 1998;26(12):1133-9.

44. Rosu-Myles M, Wolff L. p15Ink4b: dual function in myelopoiesis and inactivation in myeloid disease. Blood Cells Mol Dis. 2008;40(3):406-9.
45. Untergasser G, et al. Profiling molecular targets of TGF-beta1 in prostate fibroblast-to-myofibroblast transdifferentiation. Mech Ageing Dev. 2005;126(1):59-69.

46. Franklin DS, et al. CDK inhibitors p18(INK4c) and p27(Kip1) mediate two separate pathways to collaboratively suppress pituitary tumorigenesis. Genes Dev. 1998;12(18):2899-911.

47. $\mathrm{Yu} \mathrm{H}$, et al. Hematopoietic stem cell exhaustion impacted by p18 INK4C and p21 Cip1/Waf1 in opposite manners. Blood. 2006;107(3):1200-6.

48. Yuan Y, et al. In vivo self-renewing divisions of haematopoietic stem cells are increased in the absence of the early G1-phase inhibitor, p18INK4C. Nat Cell Biol. 2004;6(5):436-42.

49. Gao Y, et al. Small-molecule inhibitors targeting INK4 protein p18(INK4C) enhance ex vivo expansion of haematopoietic stem cells. Nat Commun. 2015;6:6328.

50. Orford KW, Scadden DT. Deconstructing stem cell self-renewal: genetic insights into cell-cycle regulation. Nat Rev Genet. 2008;9(2):115-28.

51. Xie XQ, et al. Discovery of novel INK4C small-molecule inhibitors to promote human and murine hematopoietic stem cell ex vivo expansion. Sci Rep. 2015;5:18115.

52. Gilles L, et al. P19INK4D links endomitotic arrest and megakaryocyte maturation and is regulated by AML-1. Blood. 2008;111(8):4081-91.

53. Hilpert M, et al. p19 INK4d controls hematopoietic stem cells in a cell-autonomous manner during genotoxic stress and through the microenvironment during aging. Stem Cell Reports. 2014;3(6):1085-102.

54. Brugarolas J, et al. Radiation-induced cell cycle arrest compromised by p21 deficiency. Nature. 1995;377(6549):552-7.

55. el-Deiry WS, et al. WAF1/CIP1 is induced in p53-mediated G1 arrest and apoptosis. Cancer Res. 1994;54(5):1169-74.

56. Choudhury AR, et al. Cdkn1a deletion improves stem cell function and lifespan of mice with dysfunctional telomeres without accelerating cancer formation. Nat Genet. 2007;39(1):99-105.

57. Nakayama K, et al. Mice lacking p27(Kip1) display increased body size, multiple organ hyperplasia, retinal dysplasia, and pituitary tumors. Cell. 1996;85(5):707-20.

58. Walkley CR, et al. Negative cell-cycle regulators cooperatively control self-renewal and differentiation of haematopoietic stem cells. Nat Cell Biol. 2005;7(2):172-8.

59. Zou P, et al. p57(Kip2) and p27(Kip1) cooperate to maintain hematopoietic stem cell quiescence through interactions with Hsc70. Cell Stem Cell. 2011;9(3):247-61.

60. Yoshihara $\mathrm{H}$, et al. Thrombopoietin/MPL signaling regulates hematopoietic stem cell quiescence and interaction with the osteoblastic niche. Cell Stem Cell. 2007;1(6):685-97.

61. Yamazaki S, et al. Cytokine signals modulated via lipid rafts mimic niche signals and induce hibernation in hematopoietic stem cells. EMBO J. 2006;25(15):3515-23.

62. Yamazaki S, et al. TGF-beta as a candidate bone marrow niche signal to induce hematopoietic stem cell hibernation. Blood. 2009;113(6):1250-6.

63. Matsumoto A, et al. p57 is required for quiescence and maintenance of adult hematopoietic stem cells. Cell Stem Cell. 2011;9(3):262-71.

64. van Os R, et al. A Limited role for $\mathrm{p} 21 \mathrm{Cip} 1 / \mathrm{Waf} 1$ in maintaining normal hematopoietic stem cell functioning. Stem Cells. 2007;25(4):836-43.

65. Ganter B, Fu S, Lipsick JS. D-type cyclins repress transcriptional activation by the v-Myb but not the c-Myb DNA-binding domain. EMBO J. 1998;17(1):255-68.

66. Lei W, Liu F, Ness SA. Positive and negative regulation of c-Myb by cyclin D1, cyclin-dependent kinases, and p27 Kip1. Blood. 2005;105(10):3855-61. 
67. Nakata $\mathrm{Y}$, et al. c-Myb contributes to G2/M cell cycle transition in human hematopoietic cells by direct regulation of cyclin B1 expression. Mol Cell Biol. 2007;27(6):2048-58.

68. Malaterre $\mathrm{J}$, et al. $\mathrm{c}-\mathrm{Myb}$ is required for progenitor cell homeostasis in colonic crypts. Proc Natl Acad Sci USA. 2007;104(10):3829-34.

69. Lieu YK, Reddy EP. Impaired adult myeloid progenitor CMP and GMP cell function in conditional c-myb-knockout mice. Cell Cycle. 2012;11(18):3504-12.

70. Liu D, et al. c-Myb regulates cell cycle-dependent expression of Erbin: an implication for a novel function of Erbin. PLoS One. 2012;7(8):e42903.

71. Tsai FY, et al. An early haematopoietic defect in mice lacking the transcription factor GATA-2. Nature. 1994;371(6494):221-6.

72. Ezoe S, et al. GATA-2/estrogen receptor chimera regulates cytokine-dependent growth of hematopoietic cells through accumulation of p21(WAF1) and p27(Kip1) proteins. Blood. 2002;100(10):3512-20.

73. Kitajima K, et al. GATA-2 and GATA-2/ER display opposing activities in the development and differentiation of blood progenitors. EMBO J. 2002;21(12):3060-9.

74. Persons DA, et al. Enforced expression of the GATA-2 transcription factor blocks normal hematopoiesis. Blood. 1999;93(2):488-99.

75. Hock $\mathrm{H}$, et al. Gfi-1 restricts proliferation and preserves functional integrity of haematopoietic stem cells. Nature. 2004;431(7011):1002-7.

76. Zeng $\mathrm{H}$, et al. Transcription factor Gfil regulates self-renewal and engraftment of hematopoietic stem cells. EMBO J. 2004;23(20):4116-25.

77. Antonchuk J, Sauvageau G, Humphries RK. HOXB4 overexpression mediates very rapid stem cell regeneration and competitive hematopoietic repopulation. Exp Hematol. 2001;29(9):1125-34.

78. Sauvageau G, et al. Overexpression of HOXB4 in hematopoietic cells causes the selective expansion of more primitive populations in vitro and in vivo. Genes Dev. 1995;9(14):1753-65.

79. Care A, et al. Enforced expression of HOXB7 promotes hematopoietic stem cell proliferation and myeloid-restricted progenitor differentiation. Oncogene. 1999;18(11):1993-2001.

80. Bjornsson JM, et al. Reduced proliferative capacity of hematopoietic stem cells deficient in Hoxb3 and Hoxb4. Mol Cell Biol. 2003;23(11):3872-83.

81. Chen JY, et al. Hoxb5 marks long-term haematopoietic stem cells and reveals a homogenous perivascular niche. Nature. 2016;530(7589):223-7.

82. de Graaf CA, Metcalf D. Thrombopoietin and hematopoietic stem cells. Cell Cycle. 2011;10(10):1582-9.

83. Alexander WS, et al. Deficiencies in progenitor cells of multiple hematopoietic lineages and defective megakaryocytopoiesis in mice lacking the thrombopoietic receptor c-Mpl. Blood. 1996;87(6):2162-70.
84. Qian H, et al. Critical role of thrombopoietin in maintaining adult quiescent hematopoietic stem cells. Cell Stem Cell. 2007;1(6):671-84.

85. Abkowitz JL, Chen J. Studies of c-Mpl function distinguish the replication of hematopoietic stem cells from the expansion of differentiating clones. Blood. 2007;109(12):5186-90.

86. Larsson J, Karlsson S. The role of Smad signaling in hematopoiesis. Oncogene. 2005;24(37):5676-92.

87. Chabanon A, et al. A cross-talk between stromal cell-derived factor-1 and transforming growth factor-beta controls the quiescence/cycling switch of $\mathrm{CD} 34(+)$ progenitors through FoxO3 and mammalian target of rapamycin. Stem Cells. 2008;26(12):3150-61.

88. Oshima M, Oshima H, Taketo MM. TGF-beta receptor type II deficiency results in defects of yolk sac hematopoiesis and vasculogenesis. Dev Biol. 1996;179(1):297-302.

89. Larsson J, et al. TGF-beta signaling-deficient hematopoietic stem cells have normal self-renewal and regenerative ability in vivo despite increased proliferative capacity in vitro. Blood. 2003;102(9):3129-35.

90. Challen GA, et al. Distinct hematopoietic stem cell subtypes are differentially regulated by TGF-beta1. Cell Stem Cell. 2010;6(3):265-78.

91. Zhao M, et al. Megakaryocytes maintain homeostatic quiescence and promote post-injury regeneration of hematopoietic stem cells. Nat Med. 2014;20(11):1321-6.

92. Han YC, et al. microRNA-29a induces aberrant self-renewal capacity in hematopoietic progenitors, biased myeloid development, and acute myeloid leukemia. J Exp Med. 2010;207(3):475-89.

93. $\mathrm{Hu} \mathrm{W}$, et al. miR-29a maintains mouse hematopoietic stem cell self-renewal by regulating Dnmt3a. Blood. 2015;125(14):2206-16.

94. Herrera-Merchan A, et al. miR-33-mediated downregulation of p53 controls hematopoietic stem cell self-renewal. Cell Cycle. 2010;9(16):3277-85.

95. Lechman ER, et al. Attenuation of miR-126 activity expands HSC in vivo without exhaustion. Cell Stem Cell. 2012;11(6):799-811.

96. Lechman ER, et al. miR-126 regulates distinct self-renewal outcomes in normal and malignant hematopoietic stem cells. Cancer Cell. 2016;29(2):214-28.

97. Zuckerman KS, Wicha MS. Extracellular matrix production by the adherent cells of long-term murine bone marrow cultures. Blood. 1983;61(3):540-7.

98. Varnum-Finney B, et al. The Notch ligand, Jagged-1, influences the development of primitive hematopoietic precursor cells. Blood. 1998;91(11):4084-91.

99. Wang W, et al. Notch receptor-ligand engagement maintains hematopoietic stem cell quiescence and niche retention. Stem Cells. 2015;33(7):2280-93. 